\title{
A FLEXIBLE AND ROBUST 3D COORDINATE MEASUREMENT SYSTEM BASED ON WHITE LIGHT INTERFEROMETRY FOR DEVIATION MEASUREMENTS ON SWISS-TYPE TURNING MACHINES
}

\author{
H.-W. Lahmann ${ }^{1 *}$, S. Schmalzried ${ }^{2}$, G. Ketterer ${ }^{3}$, D. Weber ${ }^{1}$, F. Welzel ${ }^{1}$, D. Jeyaraj ${ }^{1}$ \\ ${ }^{1}$ GFE-Gesellschaft für Fertigungstechnik und Entwicklung Schmalkalden e.V., Schmalkalden, Germany \\ ${ }^{2}$ HFU Furtwangen University, ITE, Tuttlingen, Germany \\ ${ }^{3}$ HFU Furtwangen University, MME, Schwenningen, Germany \\ *Corresponding author; e-mail: hw.lahmann@gfe-net.de
}

\begin{abstract}
The calibration of machine tools or industrial robots needs high precision 3-dimensional coordinate measurements. Common available systems still have the need of ambitious and time extensive processes to fulfill the requested requirements. Therefore there is a strong request for fully automated systems that allow an autonomous and easy to handle procedure for 3D volumetric deviation measurements. This exactly is the intension of the here presented 3D Coordinate Measurement System. The so called $\mu$-GPS System is based on reliable white light interferometer and robust technology. The 3D coordinates are determined via trilateration from three measured distances like it is known from the global GPS. Mainly two parts, the sensor unit (transceiver unit) including the emitter and the reference base (three cat's-eyes) which includes the three reflectors are mounted in the workspace of the machine. A model to evaluate the signals was developed which allows in combination with a 3-dimensional failure correction a 3D accuracy of more than 5 microns. This application will be the base for an exemplary and fully automated calibration process of Swiss-type turning machines. As these machines are used in high volume manufacturing for precision parts, they need to be assembled and calibrated very accurately. For the long term stability the $\mu$-GPS System can be easily assembled in the Swiss-type turning machine so that thermal deviations and abrasion can be measured and compensated in the control. The robust and easy handling of the $\mu$-GPS System later allows an expansion of the showcase to robots and other machine tools.
\end{abstract}

\section{Keywords:}

3D Measurement; Optical Measuring; Interferometry; Machine Calibration; Thermal Deviation

\section{INTRODUCTION}

The machine tool is still an important manufacturing base in the metalworking industry. Current cutting processes require robust and reliable machines. A support for machine reliability is the intrinsic machine precision. A decisive factor to achieve a high machine precision is the undergoing of a high quality machine calibration and acceptance test. However, the calibration phase after the machine assembly and set up is an unproductive time and can last more than one day depending on the machine type. In this phase, no turnover is generated with the expensive manufacturing system. Both the machine manufacturer and the machine operator are highly interested to reduce this unproductive time to a minimum. Nevertheless, a machine acceptance process is unavoidable as proof of a contractually agreed and perfectly delivered machine. At least it is necessary to prove the requirement to the standards. Unfortunately, the reality shows that the general technological progress up to now has not been transferred to the machine acceptance processes and ultra-modern machines are installed with outdated procedures. The methods and processes that were developed by Schlesinger [Schlesinger 1955] at the beginning of the 20th century are used especially in the area of geometric machine assessment. In particular, the working accuracy of a machine tool is essentially described by its geometric and kinematic behavior. Given the large number of possible causes of errors, the geometric and kinematic deviations due to the manufacturing of the machine components and their assembly must first be taken into account. Furthermore, the thermal offset due to temperature fluctuations in the machine environment must also be evaluated. The currently used geometric machine acceptance or machine assessment process is mainly carried out with the help of manual procedures that have been available in these past times. The various acceptance steps shown in the relevant standards are mainly carried out with conventional and manual measuring equipment such as a dial gauge and angle standard. 
A fully automated machine acceptance procedure is actually not conceivable due to the high assembly effort of the manual measuring equipment. The device needs to be adapted manually for the different measuring steps on the machine tool. Therefore, the need for fast and highprecision measuring technology for the acceptance process is becoming more and more important as technology advances and the drive for efficiency increases. The demand for highest quality and precision, with a simultaneous increase of automation and faster measurement speeds, increases the importance of process integrable, high-precision optoelectronic geometry measurement significantly. Accordingly, optical measuring systems, mostly laser-based interferometric methods are currently available for geometric machine measurement [Mutilba 2018]. These systems ensure accuracy, but the different steps in a complete measurement require manual and laborious set up. As a result, the requirement of a simple and flexible measuring system for fully automated application of any current system is not fulfilled.

\section{HIGH VOLUME MACHINING BASED ON SWISS-TYPE LATHES}

Machining on Swiss-type lathes has a significant share, especially for turning parts. When turning, the inherent stability of the part is of decisive importance for the part precision. In the production of rotational parts where the part length corresponds to a multiple of the part diameter, a special set up process is required to support the raw material along its length with a counter-tip and possibly one or more steady rests. A proven solution is therefore the socalled Swiss-type turning process. Developed for precision parts of watches the process is characterized by the fact that the material bar is clamped in the main spindle. The $z-$ movement in the machining area is realized by the main spindle as shown in Fig. 1. The material bar is guided backlash free in the stationary guide bush which is assembled near the tool. This means that the cutting zone is supported directly in front of the machining point close to the tool. As a result, this process makes it possible to move the material bar practically along the tools. Swiss-type Turning is used for material diameters up to $32 \mathrm{~mm}$, particularly in the small diameter range up to $20 \mathrm{~mm}$. These machines are also suitable for machining short workpieces as insert parts. On the one hand, very favorable cutting speeds can be achieved as a result of the high speeds, and on the other hand, with short workpieces, work can also be carried out directly on the headstock without the guiding bush. Swiss-type lathes are usually used in large-scale production of complex precision parts with a small diameter and a high degree of slenderness or small parts and are thus one of the most frequently used.

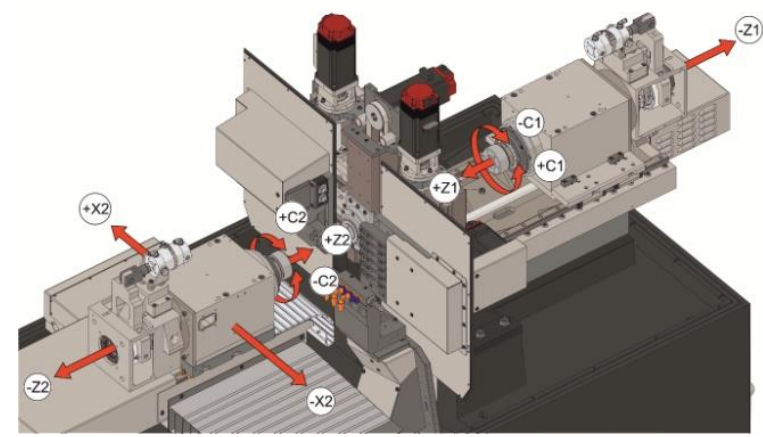

Fig. 1: Set up of a typical Swiss-type lathe.

\section{THE $\mu$-GPS SYSTEM AS A BASE FOR AN AUTOMATED MACHINE ACCEPTANCE TEST}

\subsection{History}

The $\mu$-GPS System was developed within the context of the funded ACCOMAT project from the BMBF. The idea was to use an optical 3D-based system in the control of servo mechanical axes for machine tools. The outcome of the project was that the $\mu$-GPS works reliably but the system allows just high accuracy at low measurement rates which means that the required measuring rates of more than 10 $\mathrm{kHz}$ haven't been met. On the other hand, the measuring principle was verified and the outcome was that the $\mu$-GPS is very easy to handle, contactless absolute measuring System in 3D. In a following project, a new application of the $\mu$-GPS was analyzed [Schmalzried 2007]. The intention was to use the system for applications where low measurement rates can be accepted. One of these scenarios is the calibration of machine tools, robots or automated systems.

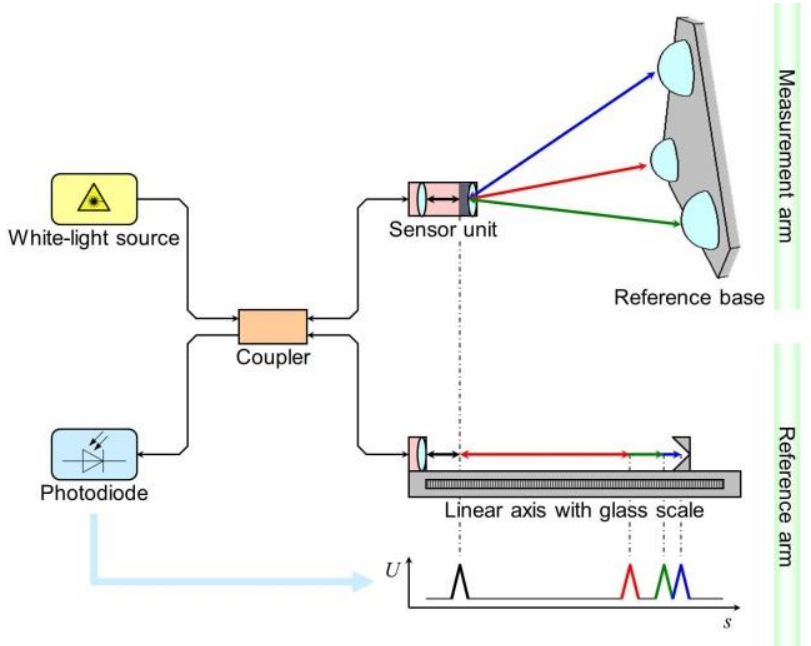

Fig. 2: Working principle of the $\mu$-GPS system.

\subsection{Working Principle}

The system is based on white light interferometry. This principle employs a light source with a small wavelength range of about $\Delta \lambda=100 \mathrm{~nm}$ and a small coherence length of $\zeta<100 \mu \mathrm{m}$. In the whole-fiber-based setup, the light is split into two beams of which one is guided to the reference arm of the interferometer and the other to the measurement arm (Fig. 2). An asymmetric beam splitter is used. In the system, it is favorable to guide $90 \%$ of the light to the transceiver unit since a lens system couples the light to free space with a total opening angle of $60^{\circ}$. The light is reflected by at least three cat's-eye retro-reflectors with an acceptance angle of about $60^{\circ}$. The reflected signal is coupled with the fiber by the lens system which also serves as a receiver and guided back to the beam splitter.

The other $10 \%$ of the signal of the light source is guided to the reference arm where the beam stays collimated. It contains basically a moveable mirror, i.e. a reference arm. Both the light reflected in the measuring arm and in the reference arm are superimposed at the beam splitter and guided to a photo diode. Due to the short coherence length, an interference signal is detected at the photo diode if the optical path in the measurement arm and reference arm is been the same. A glass scale in the reference arm helps to read the length with a micrometer resolution. Note that the reference arm is placed in an extra compartment within the control unit which is decoupled from the rest of the electronics. It is thermally stabilized and vibrations are 
damped to guarantee an accurate length measurement [Guillaume 1897].

\subsection{Position Measurement}

The small transceiver unit is usually placed at or close to the tool center point (TCP) of the machine tool and thus at the point of interest. The reference base can be placed freely in the working volume of the machine. The transceiver unit is connected with the control unit by armored fiber.

Similar to the global GPS system where the position of an object on the earth's surface is determined by three or more satellites, the position of the transceiver unit with respect to the reference base can be determined by the measured distances via trilateration (Fig. 3).

$\left(\mathrm{x}-\mathrm{u}_{\mathrm{i}}\right)^{2}+\left(\mathrm{y}-\mathrm{v}_{\mathrm{i}}\right)^{2}+\left(\mathrm{z}-\mathrm{w}_{\mathrm{i}}\right)^{2}=\mathrm{L}_{\mathrm{i}}^{2}$

Where $\mathrm{x}, \mathrm{y}, \mathrm{z}$ are the coordinates of the transceiver unit and $u_{i}, v_{i}$ and $w_{i}$ are the coordinates of the coordinates of the ith retro-reflector. The quantities $L_{i}$ denote the geometrical distance between the ith reflector and the transceiver unit. With three reflectors the position can be determined. Of course more than three reflectors can be used. In that case, the position can be determined more accurately and the overdetermined system of equations is solved using an iterative least square algorithm with estimated positions [Weule 1996]. In principle, it is also possible to determine the orientation at the TCP. Therefore, the system has to be modified to carry out measurements with four transceiver units and six reflectors.

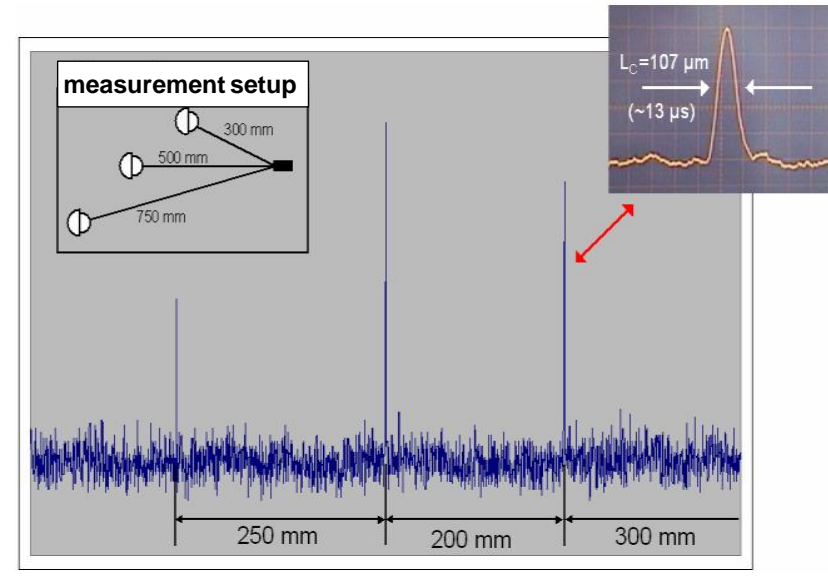

Fig. 3: Electronically processed white light signals of the cat's eyes of the reference base. Abscissa axis:

Displacement of the moveable mirror of the interferometer (Fig. 2).

\subsection{Measures to increase the accuracy of the $\mu$-GPS}

Considerable work has been done on the signal evaluation. Filters were improved to enhance the signal-to-noise ratio (SNR). For the evaluation of the signal position as a base of the machine tools the linear axes of these machines need to be highly accurate. However, the precision of the machine is much worse than a single linear axis due to errors in the assembly of the different components. As a result e.g. the squareness might not be fulfilled. Furthermore, the environmental parameters like working temperature, pressure, humidity differ from those of the standard for which the machine tool is built and certified. This leads to further deformation of the machine tool and length errors in the linear drive system.

For high precision manufacturing it is necessary to compensate these errors as far as possible. The method of choice is the so-called volumetric compensation [Sartori
1995], [Schwenke 2008]. In the following, the volumetric compensation and the state of the art methods are described. Based on that, the principle of the $\mu$-GPS System is discussed.

\subsection{Intrinsic Volumetric Compensation of the $\mu$-GPS}

For the volumetric compensation, a discrete, regular grid of positions of the tool center point (TCP) is gauged with a very precise coordinate measurement system (CMS). The results of the CMS are compared with the programmed grid and for each position an error vector is transferred to the machine control via the interface. Between the points of the grid, the error compensation vector is yielded by interpolation. To minimize errors due to the interpolation, the grid spacing must have an appropriate size. Since decreasing the grid spacing increases the measurement time, a tradeoff has to be made. To minimize the idle time and the variation of the environmental conditions, the CMS has to be fast enough. In principle, this compensation can be performed at more than one temperature to account for temperature variations that usually occur over a workday.

\section{DEVELOPMENT OF AN ACCEPTANCE TEST FOR SWISS-TYPE LATHES}

When considering the machine acceptance test with the $\mu$ GPS for Swiss-type lathes, an overview of the acceptance process that is currently established in the industry is of interest. The sequence of such a process is shown schematically in Fig. 4. The complete procedure can take up to several weeks. The design of this acceptance process is based on experience with conventional measuring equipment. As a three-dimensional method for contactless measurement is now available, a completely new acceptance process for Swiss-type lathes adapted to the $\mu$ GPS must be developed. It needs to be clarified which measurements have to be carried out one after the other and in particular which accuracy requirements are necessary for the respective measurement step. The $\mu$ GPS system should be used as an automatic system. To achieve this it is necessary to be able to carry out an automated process in accordance with a newly developed machine acceptance process that is in line with the standards. The software required for this must be able to not only record the measurements and derive tolerances from the measured values, but also act as a link between the machine's control and the $\mu$-GPS.

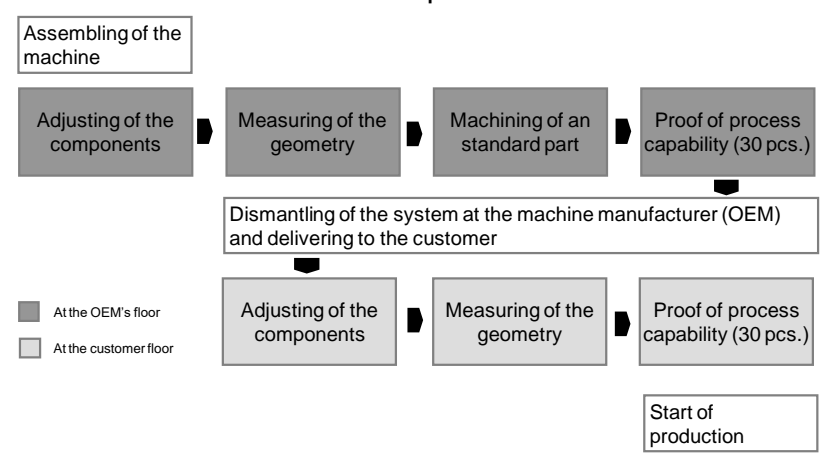

Fig. 4: Acceptance procedure until Start of Production. 


\section{INTEGRATION OF THE $\mu$-GPS SYSTEM IN THE WORKSPACE OF THE SWISS-TYPE LATHE}

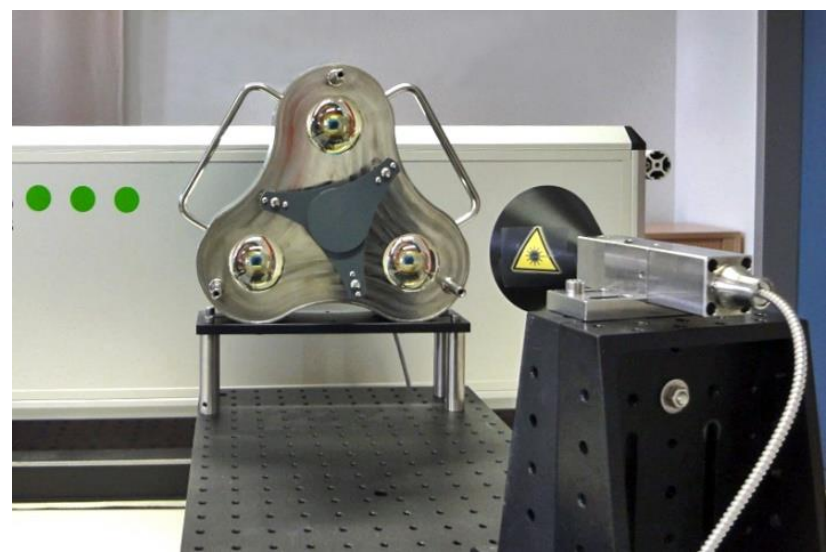

Fig. 5: Laser $\mu$-GPS measuring system showing reflector and, transceiver unit with the armed fiber cable. The measuring system is in a case in the background.

The currently realized $\mu$-GPS System with the three reflectors mounted on an equilateral triangle of $155 \mathrm{~mm}$ side length is shown in Fig. 5 . The $\mu$-GPS spans a Cartesian coordinate system as in Fig. 6 inside of the light beam.
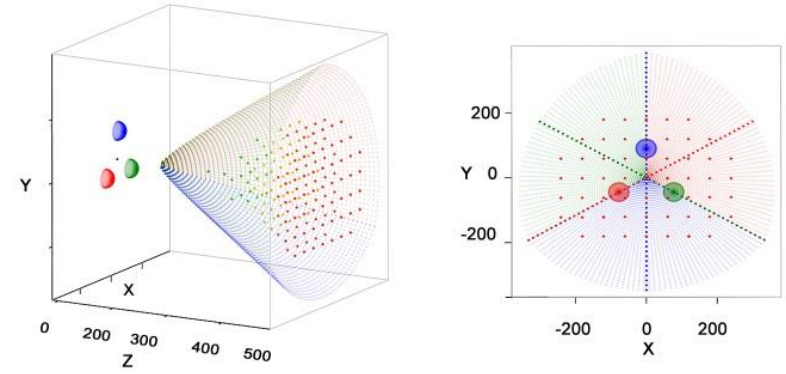

Fig. 6: Measuring volume of the $\mu$-GPS System with a 155 $\mathrm{mm}$ side length (cat's eye major radius: $40.7 \mathrm{~mm}$ ).

This system is fixed relatively by the plane of the reflector unit. For an application, this means that relative measurements can be carried out in a workspace without any alignment effort. Distances and deviations from lines can be measured relatively. If absolute measurements are to be carried out with reference to coordinate systems (machine coordinates) that are dependent on the working space, it is necessary to know the reflector base in the workspace of the machine. The reflector unit does not require any cables and can be easily integrated in the work area (Fig. 7). Only near the 6 symmetry planes of the ballreflectors of the reference unit overlapping signals occur. The transceiver unit is connected to an optical fiber. Care must be taken here that the fiber is not damaged when the transceiver unit moves.

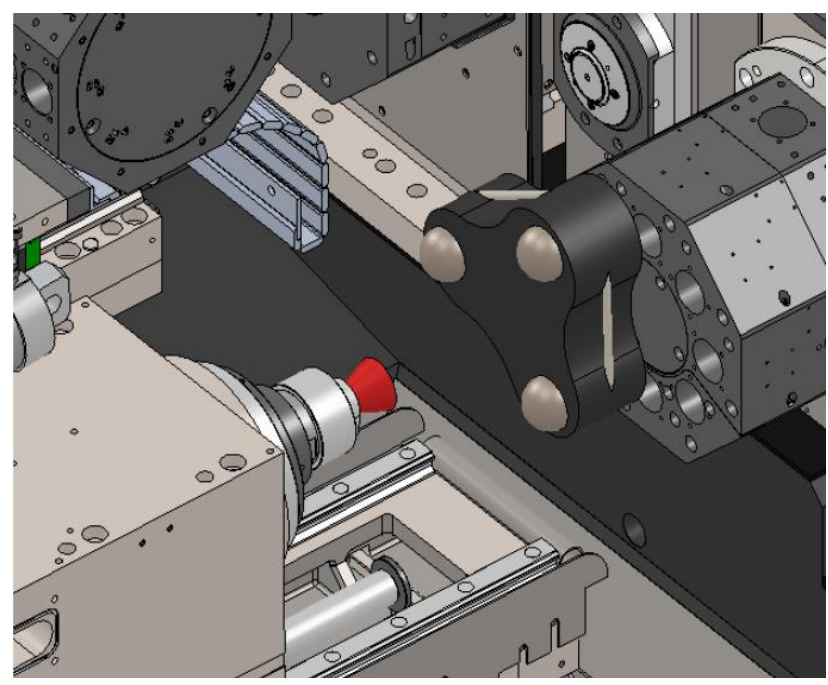

Fig. 7: Configuration of the $\mu$-GPS system in the machine. The investigation of configurations to cover the work area initially includes the definition of different variants with regard to the arrangement and technological properties of the transceiver and reflector unit in the work space so that fits in the relevant volume of the Swiss-type lathe. So now it is interesting to find by means of a mathematical simulation which arrangement is necessary to cover the working volume of the Swiss-type lathe and how this set up influences the measuring accuracy. Based on the evaluation of the workspace coverage, accuracy, economic efficiency and the technological feasibility an optimal variant can be determined as a recommendation for implementation.

5.1 Advantages through the use of a threedimensional measuring system

When using a three-dimensional measuring system, the vector from the coordinate system origin is measured to the measuring point. With one-dimensional measuring systems, each axis must be measured one after the other for the same point. Thus, with one-dimensional systems, the standard deviation in each axis is added. For the threedimensional measuring system, this means a higher standard deviation is permitted with the same accuracy. In addition, direct measurement is possible with the $\mu$-GPS. With conventional machine acceptance, several measuring or auxiliary devices are usually used for assessment (for example, angle standard and dial gauge). The use of several measuring devices and measuring aids in one measuring chain leads to increased deviations.

\subsection{Elaboration of an automated acceptance process}

The elaboration of an acceptance strategy for a Swiss-type lathe was defined in DIN ISO 230, VDI / DGQ 3441, DIN ISO 3070 .

The now planned automated measurements with the $\mu$ GPS are described in Fig. 8. This results in tremendous time saving when measuring the Swiss-type lathe in the automatically running measuring program with the new type of measuring system. 


\begin{tabular}{|c|c|c|}
\hline Notation & \multicolumn{2}{|c|}{$\begin{array}{l}\text { ASSEMBLING STEP } 5 \text { MAIN SPINDLE } \\
\text { TO COUNTER SPINDLE }\end{array}$} \\
\hline Description & \multicolumn{2}{|c|}{$\begin{array}{l}\text { Measure the center offset and the height offse } \\
\text { starting from the main spindle. }\end{array}$} \\
\hline \multicolumn{3}{|l|}{ Overview } \\
\hline Required accuracy & $\begin{array}{l}\text { Center offset max } 0.01 \mathrm{~mm} \text { Heigr } \\
0.01 \mathrm{~mm}\end{array}$ & ht offset max \\
\hline $\begin{array}{l}\text { Measurement } \\
\text { strategy with laser } \\
\mu \text {-GPS }\end{array}$ & $\begin{array}{l}\text { Evaluation of all transmission } \\
\text { units. This determines the location } \\
\text { and position of the counter spindle } \\
\text { relative to the main spindle in the } \\
X \text { and } Y \text { directions }\end{array}$ & $\begin{array}{l}\text { Laser- } \mu \text { GPS } \\
\text { Set Up: } \\
\text { A1-3 }\end{array}$ \\
\hline Expenditure of time & \multicolumn{2}{|c|}{$\begin{array}{l}\text { Iterative adjustment and measurement process } \\
\text { up to } 3 \text { hours }\end{array}$} \\
\hline $\begin{array}{l}\text { Replaced measuring } \\
\text { devices }\end{array}$ & \multicolumn{2}{|c|}{ Test pin and und dial test inidicator on device } \\
\hline
\end{tabular}

Fig. 8: Analysis of the current acceptance test.

In addition, the required compensation values will be automatically read into the compensation table of the control, so that the calibration process can run completely independently and sources of error due to structure or transmission errors are excluded.

The structure of the $\mu$-GPS will be designed in such a way that the transceiver unit and the reflector unit can be integrated temporarily in the working area of the precision lathe as shown in Fig. 9.

In case of production on a Swiss-type lathe, a double sided reflector unit is mounted in the main frame of the guide bushing. In case of controlling or readjustment, only a single sided reflector unit is mounted into the guide bushing looking to the main spindle or to the counter spindle. Mounting the transceiver unit with calibrated adapters to machine tools (WT1, WT2), the TCPs of the tools are adjustable to the main axis of the spindles by an automated process.

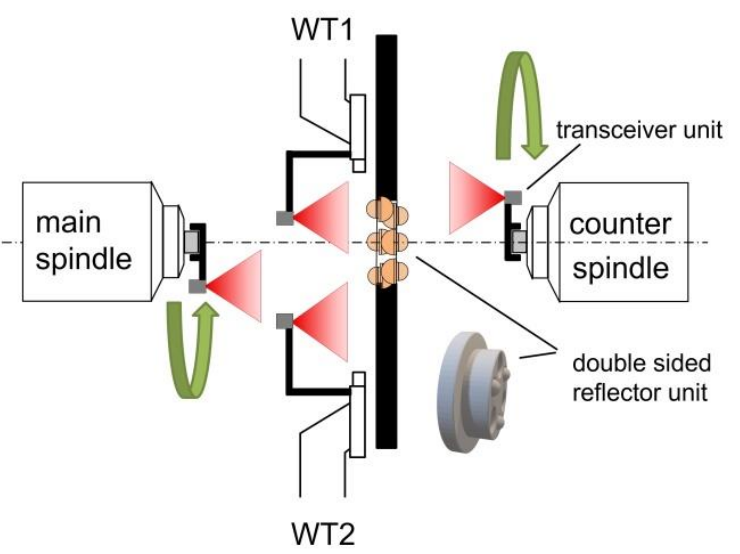

Fig. 9: Temporary mounting of a double sided reflector unit in the main frame of the guide bushing for measuring the roundness and skewness of the two spindles.

In particular, the dimensions of the work area must be taken into account, which defines the size of the reflector unit. The working area is defined by the optics of the cat's eyes, the intensity of light and by the divergence angle of the transceiver unit (approx. 60 ${ }^{\circ}$ ). The geometric behavior of the $\mu$-GPS system shown in Fig. 6 is linearly scalable.

\section{DEVIATION ESTIMATIONS FOR THE SET UP}

Besides the development of a possibly fully automated acceptance test in terms of the valid standards, the set up that fulfil a measuring step needs to be evaluated in regard to the resulting errors. Therefore, for each solution an error simulation is made. One of the possible measurements is the straightness of the main spindle relative to the counter spindle with an optical metrological space ballbar test with a fixed radius at different positions on the z-axis.

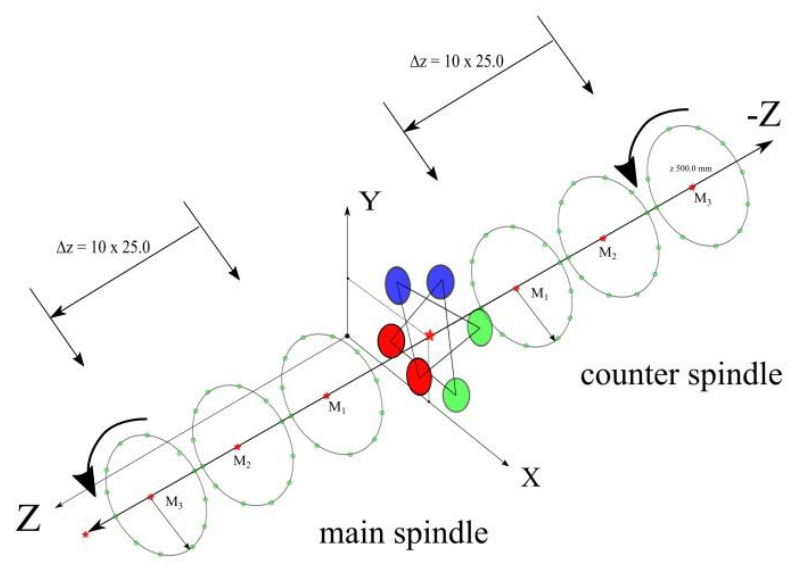

Fig. 10: Optical ballbar test with the $\mu$-GPS.

A simulation shows that while rotating the transceiver unit, the set up error of the $\mu$-GPS System is nearly eliminated.

The main advantage of this test is the insensitivity against statistical errors of the measurements as shown in Fig. 11.

The location of the turning axis of the lathe is given in the $\mu$-GPS coordinate system without any offsets. The simulation shows that the influence of the statistical errors of the measurements are reduced for adjustment or controlling. This is due to ballbar fitting by the least squares method.

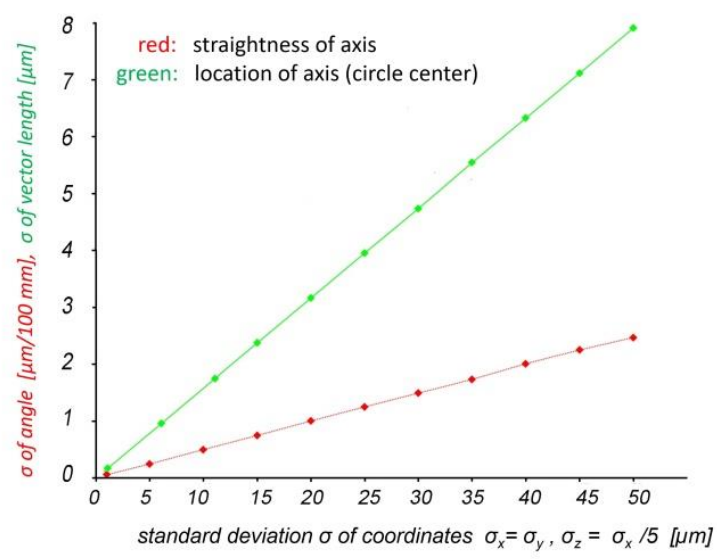

Fig. 11: Simulation of a ballbar test with the $\mu$-GPS in the actual design (13 circles, z 200-500 mm, 36 coordinates/per circle, radius: $25 \mathrm{~mm})$. Abscissa axis: errors of measurements.

The errors of the $\mu$-GPS are also scalable like the geometric behavior shown in Fig. 6. Using only points of the circles outside the 6 symmetry planes of the ball-reflectors there is no need for any electronic connection to the measuring device, because the order of the signals (Fig. 3) are known in advance.

The set up for automated measurements with application reflector unit in the Swiss-type lathe is shown in Fig. 12. 

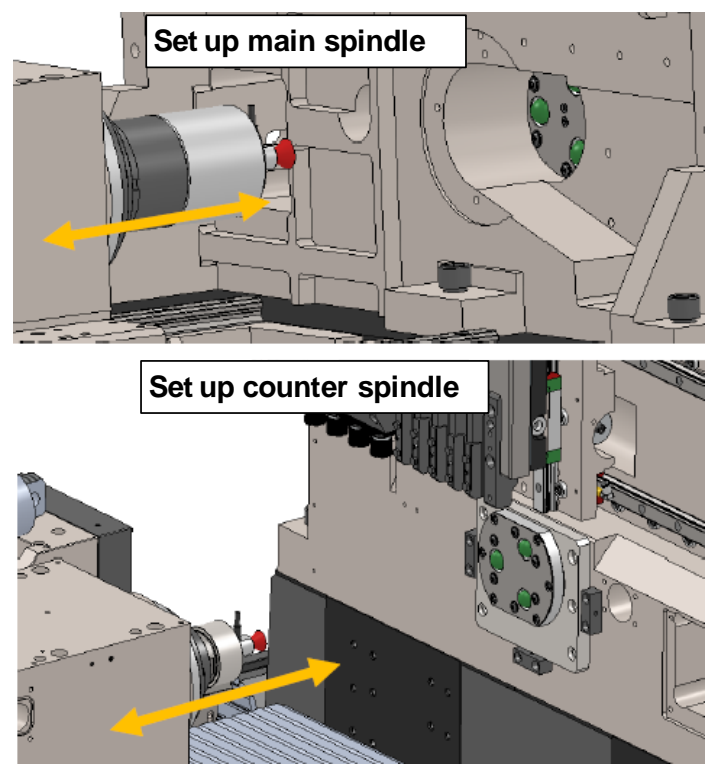

Fig. 12: Set up for automated measurements with application reflector unit in the Swiss-type lathe.

\section{AUTOMATED ACCEPTANCE TEST}

The potential of the automated acceptance test which will be realized on the basis of the $\mu$-GPS is summarized in Fig. 13. It shows a comparison of processing times (manual and $\mu$-GPS) for various steps in the procedure for an acceptance test aligned with current standards. A time saving of approx. 80 minutes will be possible especially for the Swiss-type lathe. In addition, the required compensation values will be automatically read into the compensation table of the control, so that the calibration process can run completely independently. Sources of error due to different set ups of complicated measuring devices or manual errors are excluded.

\begin{tabular}{|c|c|c|c|c|}
\hline Nr. & $\begin{array}{l}\text { Description aligned with current } \\
\text { standards }\end{array}$ & \begin{tabular}{|l} 
Required \\
accuracy
\end{tabular} & $\begin{array}{c}\text { Process } \\
\text { time } \\
\text { manual } \\
\text { [min] }\end{array}$ & $\begin{array}{c}\text { Process } \\
\text { time } \\
\mu-G P S \\
\text { (plan) } \\
\text { [min] } \\
\end{array}$ \\
\hline 1 & $\begin{array}{l}\text { Sag and angle compensation } \\
\text { Rotation on the B-axis moving } \\
\text { Y-axis }\end{array}$ & $0,01 \mathrm{~mm} / 1000 \mathrm{~mm}$ & 15 & 3 \\
\hline 2 & $\begin{array}{l}\text { Sag and angle compensation } \\
\text { Installation and alignment of } \\
\text { the devices (X-axis) } \\
\end{array}$ & $0,002 \mathrm{~mm} / 160 \mathrm{~mm}$ & 15 & 1 \\
\hline 3 & $\begin{array}{l}\text { Sag and angle compensation } \\
\text { Determining compensation } \\
\text { value } Y \text {-axis }\end{array}$ & $\begin{array}{l}0,005 \mathrm{~mm}- \\
0,01 \mathrm{~mm}\end{array}$ & 35 & 5 \\
\hline 4 & $\begin{array}{l}\text { Sag and angle compensation } \\
\text { Determining compensation } \\
\text { value } X / Y \text { interpolation } \\
\text { Alignment of the turret } \\
\end{array}$ & $0,005 \mathrm{~mm}$ & 35 & 5 \\
\hline 5 & $\begin{array}{l}\text { Sag and angle compensation } \\
\text { Alignment of the turret }\end{array}$ & $<10 \mu \mathrm{m}$ & 10 & 1 \\
\hline 6 & Backlash of the axes & $3 \mu \mathrm{m}$ & 35 & 20 \\
\hline 7 & $\begin{array}{l}\text { Center offset between the counter } \\
\text { spindle and the main spindle }\end{array}$ & $<10 \mathrm{~mm}$ & 5 & 4 \\
\hline & Align the multifunctional unit & $10 \mu \mathrm{m}$ & & 5 \\
\hline \multicolumn{3}{|c|}{ Total time required for machine acceptance manually } & $\begin{array}{l}\mathbf{1 3 0} \\
\text { (deviation } \\
20 \% \text { ) }\end{array}$ & \\
\hline \multicolumn{3}{|c|}{$\begin{array}{l}\text { Total time required for machine acceptance, 3D } \mu \text {-GPS } \\
\text { Position measuring system }\end{array}$} & & $\begin{array}{l}44 \\
\text { (deviation } \\
20 \% \text { ) } \\
\end{array}$ \\
\hline \multicolumn{3}{|c|}{ Time saved with the 3D $\mu$-GPS position measuring system [min] } & & $70-90$ \\
\hline
\end{tabular}

Fig. 13: Automated acceptance test with the $\mu$-GPS and time savings.

\section{SUMMARY}

In summary, in this paper it is discussed how flexible and robust 3D coordinate measurements can be used for the machine acceptance test for Swiss type lathes. Especially the cutting processes of Swiss-type lathes require best calibrated machines for their high accurate reliable production processes. The so called $\mu$-GPS System allows for an easy implementation and easy handling associated with a tremendously reduced time need for the complete calibration process. The basis of the time saving is the opportunity to execute most of the necessary acceptance procedures in a fully automated process.

To accomplish this specific application with the requirements of the standard acceptance procedure it was necessary to increase the intrinsic accuracy of the $\mu$-GPS. Therefore, first the hardware of the $\mu$-GPS was adapted to the workspace of a Swiss-type lathe.

In a next step, a solution for the straightness of the main and counter spindles was mathematically and metrological examined and adapted for the new application. The developments in this area are currently still in progress and promise a lot of potential for the future.

Currently, the focus of the application of the $\mu$-GPS is examined very specifically for the Swiss-type lathe. However, if the implementation is successful, future applications will be expanded to include milling machines, industrial robots and other complicated kinematics. In addition, this measuring system can also be used to measure thermal displacements in machine tools, ideally with complete retention in the machine. Research is also being carried out on this with a lower-cost version.

\section{ACKNOWLEDGMENTS}

Special thanks go to Maier and the entire Maier-GPS team for the enormous support of the project and for the possibility of scientific research.

We thank Gerald Werner (Fibotec) and the company Kematec.

\section{REFERENCES}

[Schlesinger 1955] Schlesinger, G. Prüfbuch für Werkzeugmaschinen. 6. Auflage, Verlag G.W. den Boer, Middelburg, 1955.

[Mutilba 2018] Mutilba, U., Yague-Fabra, J. A., GomezAcedao, E., Kortaberria, G., Olarra, A. Integrated multilateration for machine tools automatic verification. CIRP Annals-Manufacturing Technology 67, 2018, pp. 555558.

[Sartori 1995] Sartori, S., Zhang, GX. Geometric Error Measurement and Compensation of Machines. Annals of the CRIP, Vol.44 (2), 1995, pp. 599-609.

[Schwenke 2008] Schwenke, H., Knapp, W., Haitjema, H., Weckenmann, A., Schmitt, R., Delbressine, F. Geometric Error Measurement and Compensation of Machines - An Update. Annals of the CRIP, Vol.57, 2008, pp. 660-675.

[Schmalzried 2007] Schmalzried, S. Dreidimensionales optisches Messsystem fur eine effizientere geometrische Maschinenbeurteilung. Shaker Verlag, 2007.

[Weule 2005] Plutowsky, A., Hoeller, F., Spieweck, M. and Werner, J. A Three-Degree-of-Freedom Measurement system for Machine Tools. Proc. SPIE, vol.5638, 2005, pp. 387-394.

[Guillaume 1897] Guillaume, C.E. C.R. Acad. Sci., vol.125, 1897, pp. 235. 